\title{
COMMENTARY
}

\section{BTLA as a biomarker and mediator of sepsis- induced immunosuppression}

\author{
Edward R Sherwood ${ }^{1,2^{*}}$ and Richard S Hotchkiss ${ }^{3}$ \\ See related research by Shubin et al., http://ccforum.com/content/17/6/R276
}

\begin{abstract}
Recent research indicates that T-lymphocyte dysfunction may contribute to sepsis-associated morbidity and mortality. B and T lymphocyte attenuator (BTLA) is a co-inhibitory receptor expressed by $T$ lymphocytes and $\mathrm{B}$ lymphocytes that is important in regulating lymphocyte activation during inflammation and infection. Shubin and colleagues report that higher mean BTLA expression in critically ill patients may have value in identifying patients with infection. Further studies provide evidence that BTLA activation contributes to T-lymphocyte apoptosis during sepsis. Although this study will require follow-up and further investigation, the results advance current knowledge regarding potential mechanisms underlying sepsis-induced immunosuppression and identify BTLA as a candidate biomarker and mediator of T-cell dysfunction during sepsis.
\end{abstract}

The paper by Shubin and colleagues published in this issue of Critical Care describes expression of the immunomodulatory protein $\mathrm{B}$ and $\mathrm{T}$ lymphocyte attenuator (BTLA) on $\mathrm{CD} 4^{+} \mathrm{T}$ cells from critically ill patients with sepsis or systemic inflammatory response syndrome (SIRS) [1]. BTLA is a co-inhibitory protein that is expressed by $\mathrm{T}$ lymphocytes and interacts primarily with a tumor necrosis factor superfamily molecule termed herpes virus entry mediator [2]. Surface expression of BTLA is low on naive $\mathrm{CD}^{+} \mathrm{T}$ cells, but is rapidly upregulated following T-lymphocyte activation. Herpes virus entry mediator is constitutively expressed on dendritic

\footnotetext{
* Correspondence: edward.r.sherwood@vanderbilt.edu

'Department of Anesthesiology, Vanderbilt University Medical Center, Research Division, 1161 Medical Center Drive, Nashville, TN 37232-2520, USA

${ }^{2}$ Department of Pathology, Microbiology and Immunology, Vanderbilt University Medical Center, Research Division, 1161 Medical Center Drive, Nashville, TN 37232-2520, USA

Full list of author information is available at the end of the article
}

cells, B lymphocytes, natural killer cells, natural killer $\mathrm{T}$ cells and $\gamma \delta$ T cells. The interaction of herpes virus entry mediator with BTLA induces bidirectional signaling pathways that balance activation and inhibition to regulate T-lymphocyte activation [3]. BTLA is thus a coinhibitory receptor that, when activated, has the potential to facilitate T-cell dysfunction during sepsis and critical illness.

Recent research has raised the concept that patients with sepsis often die from persistent primary infection or development of secondary infections due to impaired adaptive antimicrobial immunity. Otto and colleagues reported that $63 \%$ of deaths in septic patients occur more than 6 days after the diagnosis of sepsis and are associated with infection by opportunistic bacteria and fungi [4]. Evidence indicates that impaired T-lymphocyte function contributes to the increased susceptibility to infection during the later phases of sepsis. Several recent reports describe upregulation of co-inhibitory receptors such as cytotoxic T-lymphocyte antigen-4, programmed cell death protein-1, and lymphocyte activation gene-3 on $\mathrm{T}$ lymphocytes from patients with sepsis [5-7]. Activation of co-inhibitory receptors may induce T-cell dysfunction, exhaustion, and anergy, with subsequent inability to adequately respond to active and subsequent secondary infections. In a large-scale postmortem study, Boomer and colleagues showed widespread Tlymphocyte apoptosis and exhaustion in patients who died during the later phases of sepsis [8]. Most of the patients that died in Boomer and colleagues' study showed evidence of ongoing infection and increased expression of co-inhibitory receptors. These findings raise the possibility that T-cell co-inhibitory receptors, and their ligands, may serve as useful biomarkers to characterize the immunological state of patients with sepsis [9]. In addition, experimental studies show that blockade of coinhibitory receptors will improve the response to opportunistic infections in the septic host $[10,11]$. The latter observations have prompted interest in utilizing co- 
inhibitory receptor blockade as a therapeutic approach to improve antimicrobial immunity during human sepsis [12].

Shubin and colleagues report higher mean cell surface BTLA expression on peripheral blood $\mathrm{CD}^{+}{ }^{+} \mathrm{T}$ cells from patients with sepsis compared with critically ill nonseptic patients. They further report higher mean surface BTLA expression on blood $\mathrm{CD}^{+}{ }^{+} \mathrm{T}$ cells from SIRS patients that developed nosocomial infections compared with SIRS patients that remained infection free. Based on their observations, the authors propose that BTLA could serve as a biomarker to identify critically ill patients that are at risk of developing nosocomial infection as well as to differentiate critically ill patients with sepsis from those with SIRS. This is an important undertaking since the identification of biomarkers that can differentiate critically ill patient populations could positively alter patient management. Examination of their results shows an absence of infection in patients with $<80 \%$ blood $\mathrm{BTLA}^{+} \mathrm{CD} 4^{+} \mathrm{T}$ cells. However, most patients in their cohort had $>80 \%$ BTLA $^{+} \mathrm{CD}^{+}{ }^{+} \mathrm{T}$ cells in their blood, regardless of whether they were infected. High BTLA expression was thus poorly predictive of the presence of infection. These observations highlight the challenges associated with identifying biomarkers with strong predictive value for differentiating infected patients from non-infected patients in the ICU. Nevertheless, their findings show that critically ill patients with $<80 \%$ BTLA ${ }^{+} \mathrm{CD} 4{ }^{+} \mathrm{T}$ cells are most probably infection free. If confirmed in large-scale studies, this information could be valuable in guiding patient management. One should note that a major difficulty with this approach will be standardizing acquisition parameters in flow cytometers from different institutions. Even with use of fluorescent bead standards, this can be a difficult challenge.

Shubin and colleagues performed further studies in mice to assess the impact of sepsis on BTLA expression and to determine the functional importance of BTLA in facilitating lymphocyte apoptosis. The mouse model of cecal ligation and puncture was used. BTLA was expressed by $>95 \%$ of blood and splenic $\mathrm{T}$ cells and $\mathrm{B}$ cells after sham and cecal ligation and puncture procedures. A small but significant increase in BTLA mean fluorescence intensity was observed for both cell populations by 72 hours after the induction of sepsis, indicating increased BTLA expression on individual cells. Further studies demonstrated significant lymphocyte apoptosis in the thymus and spleen after cecal ligation and puncture, which was attenuated in BTLA-deficient mice. These studies show an association between BTLA expression and sepsis-induced apoptosis and infer a possible cause and effect relationship. Previous studies by the group showed enhanced survival and decreased bacterial burden in BTLA-deficient mice after cecal ligation and puncture [13]. Taken together, their findings imply a functional role for BTLA in mediating sepsis-induced immunosuppression.

As noted by the investigators, the current clinical study is a small, single-institutional trial that will require large-scale follow-up to determine whether BTLA expression can serve as a useful biomarker to identify critically ill patients that are at risk of developing infection. More research is also needed to evaluate the efficacy of BTLA blockade as an approach to improve the host response to infection in vulnerable populations. Despite those limitations, the paper by Shubin and colleagues advances current knowledge regarding the impact of sepsis and critical illness on T-cell function and identifies BTLA as a potential biomarker and mediator of Tcell dysfunction in critically ill patients.

\section{Abbreviations}

BTLA: B and T lymphocyte attenuator; SIRS: Systemic inflammatory response syndrome.

\section{Competing interests}

The authors declare that they have no competing interests.

\section{Author details}

${ }^{1}$ Department of Anesthesiology, Vanderbilt University Medical Center, Research Division, 1161 Medical Center Drive, Nashville, TN 37232-2520, USA. 2Department of Pathology, Microbiology and Immunology, Vanderbilt University Medical Center, Research Division, 1161 Medical Center Drive, Nashville, TN 37232-2520, USA. ${ }^{3}$ Department of Anesthesiology, Washington University School of Medicine, 660 S. Euclid Avenue, St Louis, MO 63110, USA.

Published: 09 Dec 2013

\section{References}

1. Shubin NJ, Monaghan SF, Heffernan DS, Chung CS, Ayala A: B and T lymphocyte attenuator expression on $\mathrm{CD}^{+}{ }^{+} \mathrm{T}$-cells associates with sepsis and subsequent infections in ICU patients. Crit Care 2013, 17:276.

2. Sykulev Y: T cell receptor signaling kinetics takes the stage. Sci Signal 2010, 3:pe50.

3. Steinberg MW, Cheung TC, Ware CF: The signaling networks of the herpesvirus entry mediator (TNFRSF14) in immune regulation. Immunol Rev 2011, 244:169-187.

4. Otto GP, Sossdorf M, Claus RA, Rodel J, Menge K, Reinhart K, Bauer M, Riedemann NC: The late phase of sepsis is characterized by an increased microbiological burden and death rate. Crit Care 2011, 15:R183.

5. Boomer JS, Shuherk-Shaffer J, Hotchkiss RS, Green JM: A prospective analysis of lymphocyte phenotype and function over the course of acute sepsis. Crit Care 2012, 16:R112.

6. Guignant C, Lepape A, Huang X, Kherouf H, Denis L, Poitevin F, Malcus C, Cheron A, Allaouchiche B, Gueyffier F, Ayala A, Monneret G, Venet F: Programmed death-1 levels correlate with increased mortality, nosocomial infection and immune dysfunctions in septic shock patients. Crit Care 2011, 15:R99.

7. Zhang Y, Li J, Lou J, Zhou Y, Bo L, Zhu J, Zhu K, Wan X, Cai Z, Deng X: Upregulation of programmed death- 1 on $T$ cells and programmed death ligand-1 on monocytes in septic shock patients. Crit Care 2011, 15:R70.

8. Boomer JS, To K, Chang KC, Takasu O, Osborne DF, Walton AH, Bricker TL, Jarman SD 2nd, Kreisel D, Krupnick AS, Srivastava A, Swanson PE, Green JM, Hotchkiss RS: Immunosuppression in patients who die of sepsis and multiple organ failure. JAMA 2011, 306:2594-2605.

9. Hotchkiss RS, Monneret G, Payen D: Immunosuppression in sepsis: a novel understanding of the disorder and a new therapeutic approach. Lancet Infect Dis 2013, 13:260-268. 
10. Chang KC, Burnham CA, Compton SM, Rasche DP, Mazuski R, Smcdonough J, Unsinger J, Korman AJ, Green JM, Hotchkiss RS: Blockade of the negative co-stimulatory molecules PD-1 and CTLA-4 improves survival in primary and secondary fungal sepsis. Crit Care 2013, 17:R85.

11. Zhang Y, Zhou Y, Lou J, Li J, Bo L, Zhu K, Wan X, Deng X, Cai Z: PD-L1 blockade improves survival in experimental sepsis by inhibiting lymphocyte apoptosis and reversing monocyte dysfunction. Crit Care 2010, 14:R220.

12. Hotchkiss RS, Opal S: Immunotherapy for sepsis - a new approach against an ancient foe. N Engl J Med 2010, 363:87-89.

13. Shubin NJ, Chung CS, Heffernan DS, Irwin LR, Monaghan SF, Ayala A: BTLA expression contributes to septic morbidity and mortality by inducing innate inflammatory cell dysfunction. J Leukoc Biol 2012, 92:593-603.

\section{$10.1186 / \mathrm{cc} 13143$}

Cite this article as: Sherwood and Hotchkiss: BTLA as a biomarker and mediator of sepsis-induced immunosuppression. Critical Care 